\title{
Association between miR-199a rs74723057 and MET rs1621 polymorphisms and the risk of hepatocellular carcinoma
}

\author{
Qianqian Wang ${ }^{2, *}$, Xiangyuan $\mathbf{Y u}^{2,{ }^{*}}$, Qiang $\mathbf{L i}^{2}$, Linyuan Qin ${ }^{2}$, Shengkui Tan ${ }^{2}$, Xiaoyun \\ Zeng $^{1}$, Xiaoqiang Qiu', Bo Tang ${ }^{3}$, Junfei Jin ${ }^{3}$, Weijia Liao ${ }^{3}$, Moqin Qiu ${ }^{3}$, Lijun Tan ${ }^{3}$, \\ Gaofeng $\mathrm{He}^{3}$, Xiaomei $\mathrm{Li}^{2}$, Songqing $\mathrm{He}^{3,4}$, Hongping $\mathrm{Yu}^{1,2}$ \\ ${ }^{1}$ Department of Epidemiology and Health Statistics, Guangxi Medical University, Nanning 530021, China \\ ${ }^{2}$ Department of Epidemiology, School of Public Health, Guilin Medical University, Guilin 541004, China \\ ${ }^{3}$ Laboratory of Hepatobiliary and Pancreatic Surgery, The Affiliated Hospital of Guilin Medical University, Guilin 541001, China \\ ${ }^{4}$ Guangxi Key Laboratory of Molecular Medicine in Liver Injury and Repair, Guilin Medical University, Guilin 541001, China \\ *These authors contributed equally to this work
}

Correspondence to: Hongping Yu, email: yhp268@163.com

Songqing He, email: dr_hesongqing@163.com

Keywords: hepatocellular carcinoma, miR-199a, MET, single nucleotide polymorphism, risk

Received: April 22, 2016

Accepted: October 21, 2016

Published: November 02, 2016

\section{ABSTRACT}

MicroRNAs (miRNAs) can regulate gene expression at post-transcriptional levels, thereby influence cancer risk. The aim of the current study is to investigate association between miR-199a rs74723057 and MET rs1621 and HCC risk in 1032 HCC patients and 1060 cancer-free controls. These two SNPs were genotyped by using the Agena MassARRAY genotyping system. Odds ratio (OR) and $95 \%$ confidence interval $(95 \% \mathrm{CI})$ were calculated to assess the strength of the associations. We found that compared with the wild-type AA genotype of MET rs1621, the variant GG genotype was associated with a decreased risk for HCC $(\mathrm{OR}=0.24,95 \% \mathrm{CI}=0.06-0.96$, $P=0.043$ ). No association between miR-199a rs74723057 and HCC risk was observed. In addition, an interaction effect on HCC risk between the selected two SNPs was found. Among those who carried the CG/GG genotypes of miR-199a rs74723057, those who carried the GG genotype of MET rs1621 had a reduced risk of HCC, when compared with those who carried the AG/AA genotypes of MET rs1621 (OR $=0.15$, $95 \% \mathrm{CI}=0.03 \sim 0.73, P$ for interaction $=0.018)$. Our results suggest that MET rs1621 polymorphism, alone and combined with miR-199a rs74723057, may influence susceptibility to HCC. Further large-scale association studies and functional studies are needed to validate our findings.

\section{INTRODUCTION}

Liver cancer is one of the most common malignant tumors in the world. Hepatocellular carcinoma (HCC) accounts for $85 \%$ to $90 \%$ of the primary liver cancer. China is a country with a high incidence of liver cancer. It was estimated that approximately $50 \%$ of the new cases and death cases in world occurred in China [1]. The etiology of $\mathrm{HCC}$ is multifactorial, and chronic hepatitis B virus (HBV) infection is a major cause of HCC. However, the fact that only a small proportion of patients with chronic HBV infection eventually develop HCC suggests that genetic predisposition may play an important role in the development of HCC [2-4].
MicroRNAs (miRNAs) are small (20-25 nucleotides) non-coding RNAs, which degrade or inhibit the translation of their target messenger RNAs (mRNAs) by pairing with the $3^{\prime}$ untranslated region ( $\left.3^{\prime} \mathrm{UTR}\right)$ of mRNAs, thus regulate gene expression at post-transcriptional levels [5-7]. Compelling evidence indicates that miRNAs play an essential role in a variety of biological pathways including proliferation, differentiation, apoptosis, as well as various cancers $[8,9]$. MiR-199a is an important regulatory factor, which has the similar function as tumor suppressors. Dysregulated expression of the miR-199a-3p and miR-199a-5p has been reported in many different types of human cancers including HCC [10-14]. It has been reported that miR-199a was linked to many target 
genes, such as MET, mTOR, and DDR1 [13, 15, 16]. MET proto-oncogene is involved in the process of cell proliferation, movement, differentiation and angiogenesis by combining with hepatocyte growth factor (HGF) [17-19]. Many studies have reported that MET is overexpressed in a variety of human cancers including liver, lung, breast, and colon cancer [19-24]. Wang [24] et al. reported that transgenic mice that overexpressed MET in hepatocytes spontaneously developed HCC, and when the transgene was inactivated, tumors regressed even at advanced stages of tumor progression, indicating that MET is involved in the development and process of HCC.

Sing nucleotide polymorphisms (SNPs) in miRNAs and their target genes (miRNA-binding SNPs) might affect the expression of miRNAs or miRNA-binding interactions by influencing the miRNA-mRNA binding efficacy, thereby playing a role in the development and progression of cancers [25-27]. Previous studies have shown that miR-199a and MET are implicated in human cancers including HCC [10-14, 19-24]. However, as far as we know, no research has been reported to evaluate the effect of variants in MET and miR-199a on the risk of HCC. In this hospital-based case-control study, we performed the genotyping of miR-199a rs74723057 and MET rs1621 and assessed their associations with HCC risk in the population of South China.

\section{RESULTS}

\section{Characteristics of cases and controls}

The distributions of demographic characteristics of the study subjects are presented in Table 1. There were no significant differences in the distribution of age and gender between the cases and the controls $(P>0.05)$. However, the cases were more likely to be smokers, drinkers and HBV infection individuals $(P<0.001)$.

\section{Associations of miR-199a rs74723057 and MET rs1621with HCC risk}

Genotype distributions of the two selected SNPs in cases and controls are summarized in Table 2. The genotype distributions of the two SNPs among the controls were in HWE $(P=0.225$ for miR-199a rs74723057, $P=0.819$ for MET rs1621). Compared with individuals carrying the wild-type genotype AA of MET rs1621, those individuals carrying the variant genotype GG had a decreased risk of $\mathrm{HCC}(\mathrm{OR}=0.24,95 \% \mathrm{CI}=0.06-0.96$, $P=0.043$ ).

We then assessed whether there was a combination effect between the miR-199a rs74723057 and MET rs1621 polymorphisms on the risk of HCC (Table 3). Among those individuals who carried the variant genotype $\mathrm{CC}$ of miR199a rs74723057, we did not find a change in HCC risk for those individuals who carried the variant genotype GG of
MET rs1621 $(\mathrm{OR}=0.76,95 \% \mathrm{CI}=0.04-14.2)$. However, among those who carried the $\mathrm{CG} / \mathrm{GG}$ genotypes of miR-199a rs74723057, those who carried the GG genotype of MET rs1621 had a decreased risk of $\mathrm{HCC}(\mathrm{OR}=0.15$, $95 \% \mathrm{CI}=0.03 \sim 0.73, P$ for interaction $=0.018)$, when compared with those who carried the wild-type AG/AA genotypes of MET rs1621.

\section{DISCUSSION}

In the current case-control study with $1032 \mathrm{HCC}$ patients and 1060 cancer-free controls, we investigated whether miR-199a rs74723057 and MET rs1621 polymorphisms are associated with the risk of HCC in the population of South China. Eventually, MET rs1621 $\mathrm{A}>\mathrm{G}$ was associated with the risk of HCC was found. Compared with individuals carrying the wild-type genotype AA, those individuals carrying the variant genotype GG had a decreased risk of HCC. Furthermore, we found that the two selected SNPs had a combination effect on the risk of HCC. Among those who carried the CG/GG genotypes of miR-199a rs74723057, those who carried the variant GG genotype of MET rs1621 had a decreased risk of $\mathrm{HCC}$, when compared with those who carried the AG/AA genotypes of MET rs1621. Our results suggested that MET rs1621 polymorphism, alone and combined with miR-199a rs74723057 polymorphism, may influence susceptibility to HCC in the population of South China. To the best of our knowledge, this is the first study to investigate association between MET SNPS and $\mathrm{HCC}$ risk.

MET, a transmembrane receptor tyrosine kinase, is located at a region on chromosome $7 q 31$ [28]. It is activated by its ligand HGF, thus playing an important role as a dominant oncogene in tumor development and progression [29]. The expression of MET has been shown to be overexpressed and correlated with poor prognosis in a number of major human cancers including HCC. It has been found that compared with the surrounding normal liver tissue, MET was overexpressed in HCC, suggesting that MET may contribute to the development of HCC $[19,20]$. Ueki et al. found that HCC patients with high MET expression had a shorter 5-year survival than those with low MET expression [20]. Recently variants in MET have been reported to be associated with the prognosis of various human cancers, such as lung cancer and gastric cancer [30, 31]. Cao [30] et al. found that compared with small cell lung cancer (SCLC) patients who carried the CT/TT genotypes of MET rs41736, those SCLC patients who carried the wild-genotype $\mathrm{CC}$ tended to have a shorter progression-free survival and overall survival. This finding suggests that MET rs41736 could be considered as a prognostic marker for SCLC. Sunakawa [31] et al. found that compared with gastric cancer (GC) patients carrying the AA genotype of MET rs40239, those GC patients carrying the GG/AG genotypes of MET rs40239 
Table 1: Distributions of selected variables in HCC cases and cancer-free controls

\begin{tabular}{|c|c|c|c|}
\hline Variables & Cases $n(\%)$ & Controls $n(\%)$ & $P^{a}$ \\
\hline All patients & 1032 & 1060 & \\
\hline Age (year) & & & 0.105 \\
\hline$<=48$ & $525(50.9)$ & $577(52.4)$ & \\
\hline$>48$ & $507(49.1)$ & $483(45.6)$ & \\
\hline Sex & & & 0.642 \\
\hline males & $901(87.3)$ & $933(88.0)$ & \\
\hline females & $131(12.7)$ & $127(12.0)$ & \\
\hline Smoking status & & & $<0.0001$ \\
\hline never & $653(63.3)$ & $892(84.2)$ & \\
\hline ever & $379(36.7)$ & $168(15.8)$ & \\
\hline Alcohol use & & & $<0.0001$ \\
\hline never & $688(66.7)$ & $919(90.8)$ & \\
\hline ever & $344(33.3)$ & $141(13.3)$ & \\
\hline HBV infection & & & $<0.0001$ \\
\hline$(-)$ & $170(16.5)$ & $962(90.8)$ & \\
\hline$(+)$ & $862(83.5)$ & $98(9.2)$ & \\
\hline
\end{tabular}

Note: HBV, hepatitis B virus; HCC, hepatocellular carcinoma;

${ }^{a}$ Two-sided chi-square test for distributions between cases and controls.

had a longer disease-free survival and overall survival . In the current study, we found that the variant homozygous genotype GG of MET rs1621( A > G ) was associated with the decreased risk of HCC.

MiRNAs can inhibit the majority of mRNA transcripts by post-transcriptional regulation. Now evidence has shown that miRNAs may contribute to various physiological processes and are involved in the development of HCC [10, 32]. It has been found that miR-199a expression was downregulated in HCC tissues when compared with adjacent non tumor tissues, and lower expression of miR-199a was correlated with low survival and poor prognosis of HCC [14, 33]. It was reported that MiR-199a-3p may inhibit HepG2 cell growth and invasion, and sensitize HepG2 cells to doxorubicin treatment and to hypoxia-induced apoptosis [15]. In addition, Shen [13] et al. found that miR-199a-5p could inhibit HCC cells invasion by binding to discoidin domain receptor 1(DDR1). A number of studies have demonstrated that SNPs in miRNAs may affect miRNA function and be involved in cancer development [34-36]. For example, Zhang [35] et al. investigated the association between miRNA196a-2 rs11614913C $>$ T and $\mathrm{HCC}$ risk in a large Chinese case-control study, and they found that compared with individuals carrying the CC genotype of rs11614913, those individuals carrying the CT/ TT genotypes showed decreased risk for HCC. $\mathrm{Ma}$ [36] et al. found that compared with subjects carrying the TT genotype of miR-499 rs3746444, those subjects carrying the $\mathrm{CT} / \mathrm{CC}$ genotypes had a higher risk of HCC. In the current study, we investigated the association between miR-199a rs74723057 and the risk of HCC, but we failed to find that miR-199a rs74723057 alone was significantly associated with HCC risk. However, when we performed a combined analysis between the miR-199a rs74723057 and MET rs1621 polymorphisms on the risk of HCC, we found the combination effect of the two selected SNPs on the risk of HCC. Among those who carried CG/GG genotypes of miR-199a rs 74723057, those who carried the variant genotype GG of MET rs 1621 had a decreased risk of HCC, when compared with those who carried the AG/AA genotypes of MET rs1621. Our findings suggested that MET rs1621 polymorphism, alone and combined with miR-199a rs74723057, may influence susceptibility to HCC. MET is predicted to a direct target of miR-199a by a computational tool of SNPinfo (http:// snpinfo.niehs.nih.gov/snpinfo). It was reported that miR199a-3p can inhibit the cell cycle modulation of HCC cells by repressing the translation of MTOR and MET [15]. Furthermore, miR-199a can suppress both MET and its downstream effector ERK2, thus inhibit not only cell proliferation but also motility and invasive capabilities of tumor cells [16]. miRNA SNPs and miRNA-binding SNPs are likely to affect the expression of the target genes, and may contribute to individual susceptibility to human cancers $[34,37,38]$.

The limitations of the study should be pointed out. First, our study was a hospital-based case-control study 
Table 2: Logistic regression analysis of association between miR-199a rs74723057 and MET rs1621 genotypes on $\mathrm{HCC}$ risk

\begin{tabular}{|c|c|c|c|c|c|}
\hline Variants & Cases $n(\%)$ & Controls $n(\%)$ & $P^{a}$ & $\begin{array}{c}\text { Adjusted OR } \\
(95 \% \text { CI })^{\mathrm{b}} \\
\end{array}$ & $P^{b}$ \\
\hline \multicolumn{6}{|c|}{ miR-199a rs74723057 } \\
\hline GG & $511(49.5)$ & $534(50.38)$ & 0.743 & 1 & \\
\hline $\mathrm{CG}$ & $436(42.2)$ & $448(42.26)$ & & $1.02(0.77-1.35)$ & 0.887 \\
\hline $\mathrm{CC}$ & $85(8.2)$ & $78(7.4)$ & & $0.97(0.56-1.62)$ & 0.901 \\
\hline \multicolumn{6}{|c|}{ MET rs 1621} \\
\hline $\mathrm{AA}$ & $802(77.7)$ & $853(80.5)$ & 0.154 & 1 & \\
\hline AG & $222(21.5)$ & $195(18.4)$ & & $1.33(0.96-1.85)$ & 0.087 \\
\hline GG & $8(0.8)$ & $12(1.1)$ & & $0.24(0.06-0.96)$ & 0.043 \\
\hline
\end{tabular}

Note: HCC, hepatocellular carcinoma; OR, odds ratio; CI, confidence interval.

${ }^{a} \mathrm{Chi}$-square test of genotype distribution among cases and controls.

${ }^{\mathrm{b}}$ Adjusted for age, sex, smoking status, alcohol use and HBV infection.

with potential selection bias. Second, our study was not powered enough to detect the interaction effects. Third, our findings were not mechanically validated.

In conclusion, our findings suggest that MET rs 1621 polymorphism, alone and combined with miR-199a rs74723057, may influence susceptibility to HCC. Further large-scale association studies and functional studies are needed to validate our findings.

\section{MATERIALS AND METHODS}

\section{Study population}

All newly and histologically confirmed HCC patients were consecutively recruited from The First Affiliated Hospital of Guangxi Medical University, Affiliated Tumor Hospital of Guangxi Medical University and Affiliated Hospital of Guilin Medical University during the period from January 2007 to April 2011. All cases had not been treated with radiation, chemotherapy, or surgical therapy before enrollment in the study. Cancer-free controls were recruited from the same hospitals during the same period, which were genetically unrelated to the cases and were frequency matched to the cases on age ( \pm 5 years) and sex. All subjects were interviewed to collect demographic data and history of environmental exposure after informed consent. Individuals who had smoked more than 6 months continuously or cumulatively in their lifetimes were defined as "ever smokers" and the rest as "never smokers". Those subjects who had drunk alcoholic beverages at least once a week for more than 6 months were "defined as ever drinkers" and the rest as "never drinkers". After interview, $5 \mathrm{ml}$ of peripheral blood sample were collected from each subject. Finally, 1032 HCC cases and 1060 cancer-free controls were included in this study. This study was approved by institutional review committee board of Guilin Medical University.

\section{SNP selection and genotyping}

Candidate SNPs, MiR-199a rs74723057 and MET rs1621, were selected based on the NCBI dbSNP database (http://www.ncbi.nlm.nih.gov/projects/SNP) and NIEHS SNPinfo (http://snpinfo.niehs.nih.gov/snpinfo). SNP rs74723057 is located at the promoter region of miR-199a, which may influence miR-199a expression by altering transcriptional activity. SNP rs1621 is located in the binding sites of miR-199a in the $3^{\prime}$ untranslated region (3'UTR) of MET gene, which may affect the miR-199a binding site activity. Genomic DNA was extracted from peripheral white blood cells by phenol-chloroform extraction and stored at $-80^{\circ} \mathrm{C}$. Genotyping of miR-199a rs74723057 and MET rs1621 polymorphisms was performed by using the Agena MassARRAY genotyping system (Agena; San Diego, CA) according to the manufacturer's instructions. The primers used for PCR were listed in Table 4. Each PCR reaction mixture contained $10 \mathrm{ng}$ of genomic DNA, $0.5 \mathrm{uL} 10 \times$ PCR Buffer (with $15 \mathrm{mM} \mathrm{MgCl}_{2}$ ), 0.4 ul $25 \mathrm{mM} \mathrm{MgCl}, 0.1$ ul $25 \mathrm{mM}$ dNTPs, 1 ul 0.5 uM primer Mix, and 0.2 ul 5 U/ul Hot Star Taq polymerase. Reaction was performed at $94^{\circ} \mathrm{C}$ for $15 \mathrm{~min}$, followed by 45 cycles at $94^{\circ} \mathrm{C}$ for $20 \mathrm{~s}, 56^{\circ} \mathrm{C}$ for $30 \mathrm{~s}$, and $72^{\circ} \mathrm{C}$ for $1 \mathrm{~min}$, with a final incubation at $72^{\circ} \mathrm{C}$ for 3 min. Unincorporated dNTPs were deactivated by using $0.5 \mathrm{U}$ of shrimp alkaline phosphatase (SAP) followed by primer extension using $0.2 \mathrm{ul}$ iPLEX Buffer Plus, $0.2 \mathrm{ul}$ iPLEX Termination mix, $0.94 \mathrm{ul}$ iPLEX Extend Primer Mix, 0.041 ul iPLEX Enzyme (Agena; San Diego, CA). The extension reactions were performed at $94^{\circ} \mathrm{C}$ for $30 \mathrm{~s}$ and then $94^{\circ} \mathrm{C}$ for $5 \mathrm{~s}$, followed by 40 cycles at $52^{\circ} \mathrm{C}$ for $5 \mathrm{~s}, 5$ cycles at $80^{\circ} \mathrm{C}$ for $5 \mathrm{~s}$, with a final incubation at $72^{\circ} \mathrm{C}$ for $3 \mathrm{~min}$. Purified extension reaction products were 
Table 3: The genotype combinations of the SNP-SNP interaction in miR-199a rs74723057 and MET rs1621 with HCC risk

\begin{tabular}{lcccccc}
\hline \multicolumn{2}{c}{ Genotypes } & & Cases $\boldsymbol{n}$ & Controls $\boldsymbol{n}$ & Adjusted OR (95\% CI) & $\boldsymbol{P}^{\mathbf{b}}$ \\
\hline miR-199a rs74723057 & MET rs1621 & & & & \\
GG+CG & $\mathrm{AA}+\mathrm{AG}$ & 941 & 972 & reference & \\
& $\mathrm{GG}$ & 6 & 10 & $0.15(0.03 \sim 0.73)$ & 0.018 \\
$\mathrm{CC}$ & $\mathrm{AA}+\mathrm{AG}$ & 83 & 76 & reference & \\
& $\mathrm{GG}$ & 2 & 2 & $0.76(0.04 \sim 14.02)$ & 0.85 \\
\hline
\end{tabular}

Note: HCC, hepatocellular carcinoma; OR, odds ratio; CI, confidence interval.

${ }^{a}$ Adjusted for age, sex, smoking status, alcohol use and HBV infection

${ }^{\mathrm{b}} P$-value for interaction

Table 4: Primers used in the screening for SNPs by MassArray

\begin{tabular}{|c|c|c|c|c|}
\hline Gene & SNP & Alleles & $\operatorname{Primers}\left(5^{\prime}-3^{\prime}\right)$ & Extension Primers $\left(5^{\prime}-3^{\prime}\right)$ \\
\hline miR-199a & rs74723057 & $\mathrm{G} / \mathrm{C}$ & $\begin{array}{l}\text { ACGTTGGATGAAGCCCACTGC } \\
\text { TTCATCCTG }\end{array}$ & $\begin{array}{l}\text { ACGGTCTCCCCTTGCAAAGTCCT } \\
\text { GACA }\end{array}$ \\
\hline \multirow{3}{*}{ MET } & \multirow{3}{*}{ rs 1621} & \multirow{3}{*}{$\mathrm{A} / \mathrm{G}$} & $\begin{array}{l}\text { ACGTTGGATGACAGTGACTGT } \\
\text { GGAGAAGGC }\end{array}$ & \multirow{3}{*}{ CAACCACATGCACTATACAGTAG } \\
\hline & & & $\begin{array}{l}\text { ACGTTGGATGGTAACCTACACC } \\
\text { ACATGCAC }\end{array}$ & \\
\hline & & & $\begin{array}{l}\text { ACGTTGGATGACCCTGAGCAG } \\
\text { AACTTTGTG }\end{array}$ & \\
\hline
\end{tabular}

spotted onto a 384-well Spectro CHIPs and measured by using the platform MALDI-TOF mass spectrometry within the Agena MassARRAY system. Genotype calling was performed in real time with MassARRAY Typer software version 4.0 and analyzed by using the MassARRAY Typer software version 4.0 .

\section{Statistical analysis}

Distributions of genotype frequencies in controls were tested by a chi-square goodness-of-fit test for HardyWeinberg equilibrium. A chi-square test was performed to make a comparison of the categorical variables, such as age, sex, smoking status, alcohol use, and HBV infection. The associations of each SNP with the risk of HCC were estimated by calculating the odds ratios (ORs) and their 95\% confidence intervals (CIs) from multivariate logistic regression models. All analyses were two sided with a statistical significance set at a $P$ value of $<0.05$. All statistical analyses were performed using SPSS software, version 18.0 (SPSS Institute, Chicago, IL).

\section{ACKNOWLEDGMENTS AND FUNDING}

This study was supported by the National Natural Science Foundation of China (No. 81460516 and No. 81660567), Science Foundation of Guangxi Department of Education of China (No. ZD2014093 and No. 0300JG2014090ZZ), Natural Science Foundation of Guangxi Province of China (No. 2015GXNSFCB139007 and No. 2015GXNSFFA139004).

\section{CONFLICTS OF INTEREST}

None declared.

\section{REFERENCES}

1. Jemal A, Bray F, Center MM, Ferlay J, Ward E, Forman D. Global cancer statistics. CA Cancer J Clin. 2011; 61:69-90.

2. Yu MC, Yuan JM. Environmental factors and risk for hepatocellular carcinoma. Gastroenterology. 2004; 127:S72-78.

3. Nault JC, Zucman-Rossi J. Genetics of hepatobiliary carcinogenesis. Semin Liver Dis. 2011; 31:173-187.

4. Fan Y, Hu D, Feng B, Wang W. The NQO1 C609T polymorphism and hepatocellular carcinoma risk. Tumour Biol. 2014; 35:7343-7350.

5. Lai EC. Micro RNAs are complementary to 3' UTR sequence motifs that mediate negative post-transcriptional regulation. Nat Genet. 2002; 30:363-364.

6. Friedman RC, Farh KK, Burge CB, Bartel DP. Most mammalian mRNAs are conserved targets of microRNAs. Genome Res. 2009; 19:92-105. 
7. Bartel DP. MicroRNAs: target recognition and regulatory functions. Cell. 2009; 136:215-233.

8. Calin GA, Croce CM. MicroRNA signatures in human cancers. Nat Rev Cancer. 2006; 6:857-866.

9. Tsuchiya S, Okuno Y, Tsujimoto G. MicroRNA: biogenetic and functional mechanisms and involvements in cell differentiation and cancer. J Pharmacol Sci. 2006; 101:267-270.

10. Jiang J, Gusev Y, Aderca I, Mettler TA, Nagorney DM, Brackett DJ, Roberts LR, Schmittgen TD. Association of MicroRNA expression in hepatocellular carcinomas with hepatitis infection, cirrhosis, and patient survival. Clin Cancer Res. 2008; 14:419-427.

11. Ichimi T, Enokida H, Okuno Y, Kunimoto R, Chiyomaru T, Kawamoto K, Kawahara K, Toki K, Kawakami K, Nishiyama K, Tsujimoto G, Nakagawa M, Seki N. Identification of novel microRNA targets based on microRNA signatures in bladder cancer. Int J Cancer. 2009; 125:345-352.

12. Iorio MV, Visone R, Di Leva G, Donati V, Petrocca F, Casalini P, Taccioli C, Volinia S, Liu CG, Alder H, Calin GA, Menard S, Croce CM. MicroRNA signatures in human ovarian cancer. Cancer Res. 2007; 67:8699-8707.

13. Shen Q, Cicinnati VR, Zhang $\mathrm{X}$, Iacob S, Weber F, Sotiropoulos GC, Radtke A, Lu M, Paul A, Gerken G, Beckebaum S. Role of microRNA-199a-5p and discoidin domain receptor 1 in human hepatocellular carcinoma invasion. Mol Cancer. 2010; 9:227.

14. Hou J, Lin L, Zhou W, Wang Z, Ding G, Dong Q, Qin L, Wu X, Zheng Y, Yang Y, Tian W, Zhang Q, Wang C et al. Identification of miRNomes in human liver and hepatocellular carcinoma reveals miR-199a/b-3p as therapeutic target for hepatocellular carcinoma. Cancer Cell. 2011; 19:232-243.

15. Fornari F, Milazzo M, Chieco P, Negrini M, Calin GA, Grazi GL, Pollutri D, Croce CM, Bolondi L, Gramantieri L. MiR-199a-3p regulates mTOR and c-Met to influence the doxorubicin sensitivity of human hepatocarcinoma cells. Cancer Res. 2010; 70:5184-5193.

16. Kim S, Lee UJ, Kim MN, Lee EJ, Kim JY, Lee MY, Choung S, Kim YJ, Choi YC. MicroRNA miR-199a* regulates the MET proto-oncogene and the downstream extracellular signal-regulated kinase 2 (ERK2). J Biol Chem. 2008; 283:18158-18166.

17. Ma PC, Maulik G, Christensen J, Salgia R. c-Met: structure, functions and potential for therapeutic inhibition. Cancer Metastasis Rev. 2003; 22:309-325.

18. Trusolino L, Bertotti A, Comoglio PM. MET signalling: principles and functions in development, organ regeneration and cancer. Nat Rev Mol Cell Biol. 2010; 11:834-848.

19. Suzuki K, Hayashi N, Yamada Y, Yoshihara H, Miyamoto Y, Ito $\mathrm{Y}$, Ito $\mathrm{T}$, Katayama $\mathrm{K}$, Sasaki $\mathrm{Y}$, Ito $\mathrm{A}$ and et al. Expression of the c-met protooncogene in human hepatocellular carcinoma. Hepatology. 1994; 20:1231-1236.
20. Ueki T, Fujimoto J, Suzuki T, Yamamoto H, Okamoto E. Expression of hepatocyte growth factor and its receptor c-met proto-oncogene in hepatocellular carcinoma. Hepatology. 1997; 25:862-866.

21. Nishimura Y, Hyuga S, Takiguchi S, Hyuga M, Itoh K, Hanawa T. Ephedrae herba stimulates hepatocyte growth factor-induced MET endocytosis and downregulation via early/late endocytic pathways in gefitinib-resistant human lung cancer cells. Int J Oncol. 2016; 48:1895-1906.

22. Ren X, Yuan L, Shen S, Wu H, Lu J, Liang Z. c-Met and ERbeta expression differences in basal-like and non-basallike triple-negative breast cancer. Tumour Biol. 2016; 37:11385-11395.

23. Gao W, Bing X, Li M, Yang Z, Li Y, Chen H. Study of critical role of c-Met and its inhibitor SU11274 in colorectal carcinoma. Med Oncol. 2013; 30:546.

24. Wang R, Ferrell LD, Faouzi S, Maher JJ, Bishop JM. Activation of the Met receptor by cell attachment induces and sustains hepatocellular carcinomas in transgenic mice. J Cell Biol. 2001; 153:1023-1034.

25. Wu M, Jolicoeur N, Li Z, Zhang L, Fortin Y, L'Abbe D, Yu Z, Shen SH. Genetic variations of microRNAs in human cancer and their effects on the expression of miRNAs. Carcinogenesis. 2008; 29:1710-1716.

26. Yu Z, Li Z, Jolicoeur N, Zhang L, Fortin Y, Wang E, Wu M, Shen SH. Aberrant allele frequencies of the SNPs located in microRNA target sites are potentially associated with human cancers. Nucleic Acids Res. 2007; 35:4535-4541.

27. Jazdzewski K, Murray EL, Franssila K, Jarzab B, Schoenberg DR and de la Chapelle A. Common SNP in premiR-146a decreases mature miR expression and predisposes to papillary thyroid carcinoma. Proceedings of the National Proc Natl Acad Sci USA. 2008; 105:7269-7274.

28. Lin JC, Naujokas M, Zhu H, Nolet S, Park M. Intron-exon structure of the MET gene and cloning of an alternativelyspliced Met isoform reveals frequent exon-skipping of a single large internal exon. Oncogene. 1998; 16:833-842.

29. Sattler M, Salgia R. c-Met and hepatocyte growth factor: potential as novel targets in cancer therapy. Curr Oncol Rep. 2007; 9:102-108.

30. Cao X, Hong X, Jia X, Zhang L, Chen G. Single-nucleotide polymorphism rs41736 located in MET was significantly associated with prognosis of small cell lung cancer patients. Med Oncol. 2014; 31:333.

31. Sunakawa Y, Wakatsuki T, Yang D, Zhang W, Ning $Y$, Stintzing S, Stremitzer S, Yamauchi S, Sebio A, El-khoueiry R, Iqbal S, Barzi A, Gerger A, Stotz M, Azuma M, Watanabe M, et al. Prognostic impact of the c-MET polymorphism on the clinical outcome in locoregional gastric cancer patients. Pharmacogenet Genomics. 2014; 24:588-596.

32. Murakami Y, Yasuda T, Saigo K, Urashima T, Toyoda H, Okanoue T, Shimotohno K. Comprehensive analysis of microRNA expression patterns in hepatocellular carcinoma and non-tumorous tissues. Oncogene. 2006; 25:2537-2545. 
33. Song J, Gao L, Yang G, Tang S, Xie H, Wang Y, Wang J, Zhang Y, Jin J, Gou Y, Yang Z, Chen Z, Wu K et al. MiR-199a regulates cell proliferation and survival by targeting FZD7. PloS one. 2014; 9:e110074.

34. Ryan BM, Robles AI, Harris CC. Genetic variation in microRNA networks: the implications for cancer research. Nat Rev Cancer. 2010; 10:389-402.

35. Zhang J, Wang R, Ma YY, Chen LQ, Jin BH, Yu H, Wang JC, Gao CF, Liu J. Association between single nucleotide polymorphisms in miRNA196a-2 and miRNA146a and susceptibility to hepatocellular carcinoma in a Chinese population. Asian Pac J Cancer Prev. 2013; 14:6427-6431.

36. Ma Y, Wang R, Zhang J, Li W, Gao C, Liu J, Wang J. Identification of miR-423 and miR-499 polymorphisms on affecting the risk of hepatocellular carcinoma in a large-scale population. Genet Test Mol Biomarkers. 2014; 18:516-524.

37. Teo MT, Landi D, Taylor CF, Elliott F, Vaslin L, Cox DG, Hall J, Landi S, Bishop DT, Kiltie AE. The role of microRNA-binding site polymorphisms in DNA repair genes as risk factors for bladder cancer and breast cancer and their impact on radiotherapy outcomes. Carcinogenesis. 2012; 33:581-586.

38. Naccarati A, Pardini B, Stefano L, Landi D, Slyskova J, Novotny J, Levy M, Polakova V, Lipska L, Vodicka P. Polymorphisms in miRNA-binding sites of nucleotide excision repair genes and colorectal cancer risk. Carcinogenesis. 2012; 33:1346-1351. 